\title{
Masking effect of heat dissipation on the current-voltage characteristics of a mesoscopic superconducting sample with leads
}

\author{
D. Y. Vodolazov ${ }^{1,2}$ and F. M. Peeters ${ }^{1, *}$ \\ ${ }^{1}$ Departement Fysica, Universiteit Antwerpen (Campus Drie Eiken), B-2610 Antwerpen, Belgium \\ ${ }^{2}$ Institute for Physics of Microstructures, Russian Academy of Sciences, 603950, Nizhny Novgorod, GSP-105, Russia \\ M. Morelle and V. V. Moshchalkov \\ Nanoscale Superconductivity and Magnetism Group, Laboratory for Solid State Physics and Magnetism, K. U. Leuven, \\ Celestijnenlaan 200D, B-3001 Leuven, Belgium
}

(Received 23 September 2004; revised manuscript received 3 January 2005; published 9 May 2005)

\begin{abstract}
A theoretical analysis based on a numerical solution of the coupled time-dependent Ginzburg-Landau and heat dissipation equations shows a strong dependence of the critical currents on the applied magnetic field in a mesoscopic square with attached contacts. In agreement with experiment we found hysteresis which are caused by a strong heat dissipation in the sample at currents close to the depairing Ginzburg-Landau current and/or the dynamics of the superconducting condensate. The theoretically obtained nonmonotonous dependence of the switching current (from superconducting to the resistive state) on the applied magnetic field, arising from the changes in the vorticity, agrees quantitatively with the experimental data. Our results show that heat dissipation leads to an increase of the hysteresis in the current-voltage characteristic and hence masks the actual dynamics of the superconducting condensate.
\end{abstract}

DOI: 10.1103/PhysRevB.71.184502

PACS number(s): 74.25.Op, 74.20.De, 73.23.-b

\section{INTRODUCTION}

After the discovery of the steplike features in the currentvoltage characteristics of superconducting whiskers ${ }^{1}$ and the explanation of this effect through the nucleation of phase slip centers $^{2,3}$ this phenomenon attracted a lot of attention from several experimental and theoretical groups (see Refs. 4 and 5 for reviews). This effect is a consequence of the nontrivial dynamics of the superconducting condensate and the normal quasiparticles near the phase slip center. ${ }^{2,4,5}$ During the phase slip process the gap goes periodically in time to zero in one point along the sample and it creates an excess of quasiparticles (electronlike and holelike) near this region. Due to the relatively large time of relaxation of nonequilibrium quasiparticles in the superconductor they may diffuse on a distance much larger than the coherence length (the size of suppression of the gap). ${ }^{6}$ It results in the existence of a nonzero electrical field (as a response to the gradient of chemical potential of nonequilibrium quasiparticles) and a finite timedependent voltage drop near the phase slip center. Unfortunately, strong heat dissipation masks this effect at temperatures far from the critical temperature ${ }^{2} T_{c}$ which prohibited the study of this effect in full details.

Recently, this subject was revisited because new experimental techniques were developed which made it possible to prepare samples with low resistance ${ }^{7}$ (i.e., diminishing the heating effects at low temperatures) or by using pulsed techniques. $^{8,9}$ The existence of phase slip centers or lines was confirmed in high-temperature superconductors ${ }^{8}$ and they were found to lead to $\mathrm{S}$-shaped $I-V$ characteristics in the voltage driven regime. ${ }^{10}$ Recently phase slip lines were directly observed ${ }^{11}$ in low temperature superconducting stripes. In Refs. 12 and 13 a new type of vortex dynamics (so called vortex channelling ${ }^{13}$ or the appearance of "kinematical" vortices $^{12}$ ) was proposed which, in our opinion, is the "bridge" between slow vortex motion and the fast phase slip line regime. Furthermore, in Ref. 14 the experimental observation of quantum phase slips was claimed.

In this paper we present experimental results on the current-voltage characteristics of a mesoscopic superconducting square with leads. In this geometry, the contacts play a crucial role (see Fig. 1) because the current density is maximal in them. Therefore we cannot consider those contacts in equilibrium. This is essentially different from previous work on superconducting film or bridge attached to "massive" superconducting "banks" where it was possible to assume the contacts in equilibrium. At low magnetic fields phase slip centers will appear in our sample in the narrowest places, where the current density is maximal. Because of inevitable heat dissipation the sample can transit locally to the normal state (for weak heat transfer) or to the superconducting resistive state (for strong heat transfer). In both cases the $I-V$

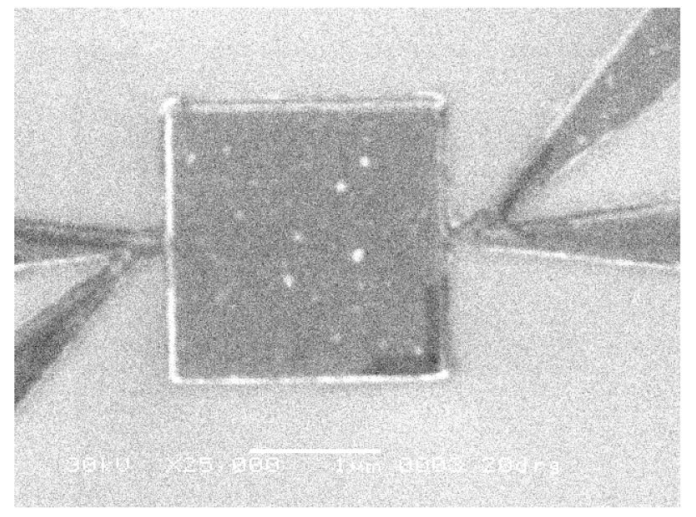

FIG. 1. SEM micrograph of an $\mathrm{Al}$ square with lateral dimension of $2 \mu \mathrm{m}$. 

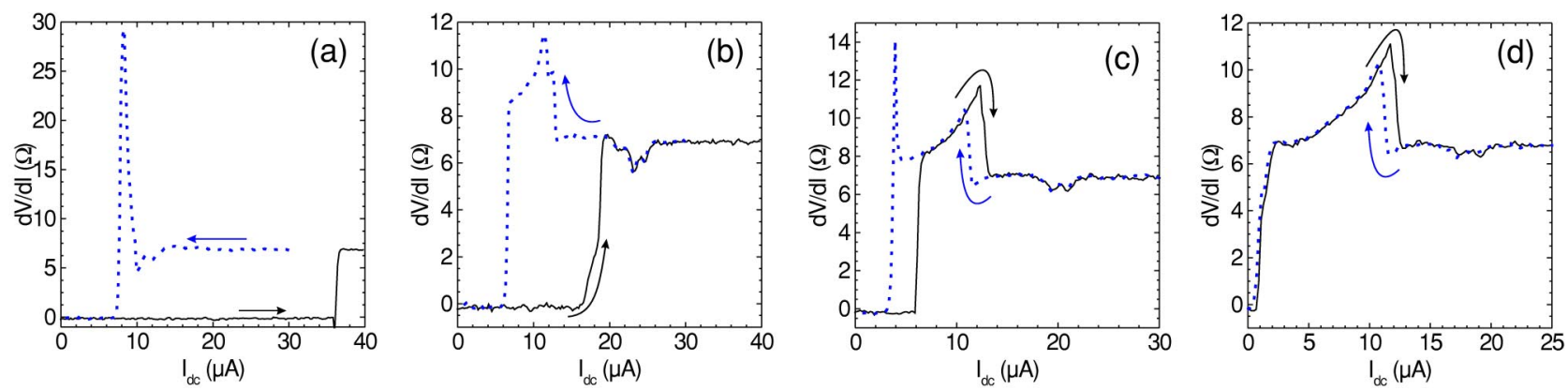

FIG. 2. (Color online) Measured $d V / d I$ as a function of the dc current at (a) $\mu_{0} H=0 \mathrm{mT}$, (b) $\mu_{0} H=3.2 \mathrm{mT}$, (c) $\mu_{0} H=4.2 \mathrm{mT}$, and (d) $\mu_{0} H=5 \mathrm{mT}$ measured at $T=1.100 \mathrm{~K}$. The full black and dashed blue lines are for increasing and decreasing current, respectively.

characteristics are hysteretic due to heat dissipation and/or the dynamics of the superconducting condensate. Additional complications come from the effect of the magnetic field induced currents in the square on the phase slip process in the contacts. This makes our system different and to our knowledge this situation was not studied before either experimentally or theoretically.

The paper is organized as follows. In Sec. II we present our experimental results and in Sec. III we give their interpretation on the basis of a solution of the time-dependent Ginzburg-Landau equations coupled with the equation for heat dissipation. Our conclusions are given in Sec. V.

\section{EXPERIMENT}

In Fig. 1 a SEM micrograph of our superconducting square made from Al using $e$-beam lithography is shown. The coherence length determined from a macroscopic coevaporated sample was found to be $\xi(0)=156 \mathrm{~nm}$. The thickness was $39 \mathrm{~nm}$ found from AFM and from x-ray measurements. Wedge shaped contacts with an opening angle of $\Gamma$ $=15^{\circ}$ were used. This shape was used to minimize the effect of the contacts on the superconducting properties of the square. ${ }^{15,16}$

The experimental $I-V$ characteristics are obtained by superimposing a small ac current $(0.1 \mu \mathrm{A}$ rms $)$ to a dc current $I_{d c}$. The ac differential resistance is measured with a EG\&G PAR 124A lock-in amplifier. The dc current is swept from negative to positive value. In order to ensure that the sample is in the normal state a high dc current is sent through the sample prior to the current sweep. Such sweeps are repeated for different magnetic fields.

In Fig. 2 the differential resistance $d V / d I\left(I_{d c}\right)$ is shown for four values of the magnetic fields. A clear hysteretic behavior is observed. When starting in the normal state and decreasing the current, the sample remains in a resistive state up to low currents. This resistive state is not the normal state anymore since a nonconstant differential resistance is observed. When starting from the superconducting state and gradually increasing the current a nonresistive state is observed up to high currents. Contrary to the transition seen at negative currents, a sharp transition from the nonresistive to the normal state is measured. While in the negative part the transition to the nonresistive state is accompanied by the appearance of a sharp peak, this is not observed for positive currents. This can be explained by our measuring technique and by the observed hysteretic behavior. When the transition occurs, the sample remains in the resistive state even when decreasing slightly the current so that the measured ac voltage will either reflect the nonresistive or the resistive state, but not the transition. Above $1.2 \mathrm{mT}$, when the first vortex enters the sample, the transition to the resistive state is preceded by a small increase of the differential resistance [see Fig. 2(b)]. The shape of this part strongly depends on the vorticity of the sample suggesting a dissipation caused by vortex motion. At high magnetic fields [see arrows in Fig. 2(b)], small symmetric features are observed at high positive and negative currents.

In order to study the magnetic field dependence in more details a contour plot of the differential resistance is shown in Fig. 3 as a function of the dc current and the magnetic field. The uniform gray (blue) areas at the left and the right correspond to the normal state and the white area in the middle to the nonresistive state. Oscillations are observed for the transition to the resistive state for positive currents with cusps each time the vorticity is changed [see arrows in Fig. 3(b)]. The dissipation caused by vortex motion, which was already discussed, can also be seen from the contour plot [light gray (green) area in Fig. 3]. The dissipation is only observed for a finite vorticity. At the transition from vorticity $L=1$ to $L=2$ at $\mu_{0} H=2.05 \mathrm{mT}$, the onset of the dissipation seems to be continuous, but the dissipation increases more rapidly with increasing current when increasing the vorticity as can also be seen from Fig. 2(b).

Beginning at $\pm 2.5 \mathrm{mT}$ small symmetric features are seen at high current [white (light blue) lines and the dark gray (dark blue) area in Figs. 3(a) and 3(b)]. These features are visible up to $\pm 20 \mathrm{mT}$ and can thus not be arising from the square since the square has a critical field $H_{c 3}^{*}=6 \mathrm{mT}$. It can only be generated by the contacts which have a higher critical field since a superconducting/normal boundary with a sharp angle increases the critical field. ${ }^{17,18}$

Above $\pm 4.5 \mathrm{mT}$ no hysteretic behavior is seen. All these features are perfectly reproduced on a different sample and are not dependent on the measuring conditions (sweep rate, amplitude of ac current, temperature). The same behavior is seen when fixing the applied dc current and sweeping the magnetic field: when the square is in the normal state, the sample remains in a resistive state up to low magnetic fields 

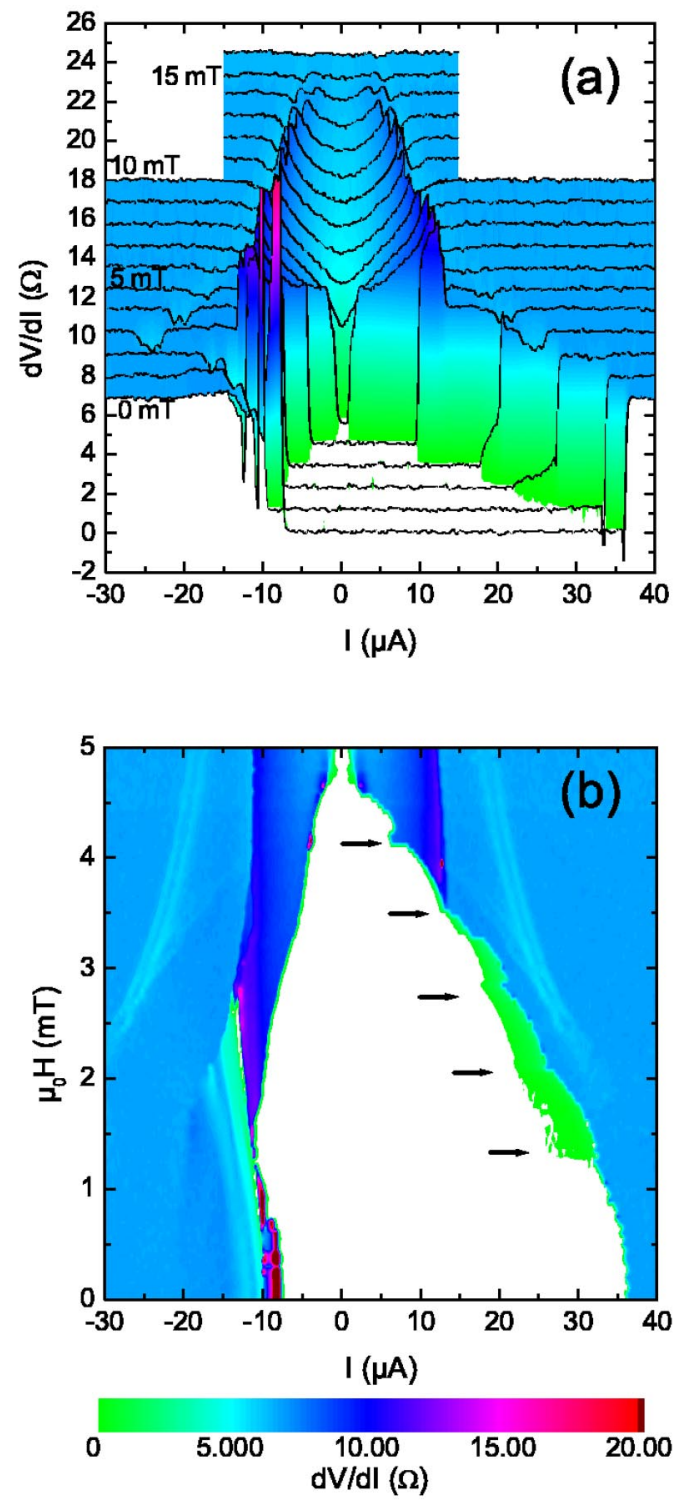

FIG. 3. (Color online) (a) Measured differential resistance $d V / d I$ as a function of the dc current for different magnetic fields measured at $T=1.100 \mathrm{~K}$. The curves for nonzero field are shifted for clarity. A color map surface is given for comparison with (b). (b) Contour plot of the differential resistance $d V / d I$ as a function of dc current and magnetic field measured at $T=1.100 \mathrm{~K}$ for low magnetic fields. The arrows indicate the points where the vorticity changes.

while when starting from the nonresistive state and increasing the magnetic field the resistive state is reached only at higher magnetic fields.

\section{THEORY}

To understand the experimental results we studied the current-voltage characteristics of two-dimensional superconductors using the generalized time-dependent GinzburgLandau (TDGL) equation ${ }^{19}$

$$
\begin{gathered}
\frac{u}{\sqrt{1+\gamma^{2}|\psi|^{2}}}\left(\frac{\partial}{\partial t}+i \varphi+\frac{\gamma^{2}}{2} \frac{\partial|\psi|^{2}}{\partial t}\right) \psi \\
=(\boldsymbol{\nabla}-i \mathbf{A})^{2} \psi+\left(1-T-|\psi|^{2}\right) \psi,
\end{gathered}
$$

where the parameter $\gamma=2 \tau_{E} \Delta_{0} / \hbar$ is the product of the inelastic collision time $\tau_{E}$ for electron-phonon scattering and $\Delta_{0}$ $=4 k_{B} T_{c} u^{1 / 2} / \pi$ is the value of the gap at $T=0$ which follows from Gor'kov's derivation ${ }^{20}$ of the Ginzburg-Landau equations.

This equation should be supplemented with the equation for the electrostatic potential

$$
\Delta \varphi=\operatorname{div}\left(\operatorname{Im}\left[\psi^{*}(\boldsymbol{\nabla}-i \mathbf{A}) \psi\right]\right),
$$

which is nothing else than the condition for the conservation of the total current in the sample, i.e., div $\mathbf{j}=0$. In Eqs. (1) and (2) all the physical quantities (order parameter $\psi$ $=|\psi| e^{i \phi}$, vector potential $\mathbf{A}$ and electrostatical potential $\left.\varphi\right)$ are measured in dimensionless units: temperature in units of the critical temperature $T_{c}$, the vector potential $\mathbf{A}$ and the momentum of the superconducting condensate $\mathbf{p}=\boldsymbol{\nabla} \phi-\mathbf{A}$ are scaled in units $\Phi_{0} /[2 \pi \xi(0)]$ (where $\Phi_{0}$ is the quantum of magnetic flux), the order parameter in units of $\Delta_{0}$ and the coordinates are in units of the coherence length $\xi(0)$ $=\left(8 k_{B} T_{c} / \pi \hbar D\right)^{-1 / 2}$. In these units the magnetic field is scaled with $H_{c 2}=\Phi_{0} / 2 \pi \xi(0)^{2}$ and the current density with $j_{0}$ $=\sigma_{n} \hbar / 2 e \tau_{G L}(0) \xi(0)$. Time is scaled in units of the GinzburgLandau relaxation time $\tau_{G L}(0)=\pi \hbar / 8 k_{B} T_{c} u$, the electrostatic potential $(\varphi)$ is in units of $\varphi_{0}=\hbar / 2 e \tau_{G L}(0)\left(\sigma_{n}\right.$ is the normalstate conductivity, and $D$ is the diffusion constant). The parameter $u$ is equal to 5.79 in accordance with Ref. 19 and we used $\gamma=40$. We put $\mathbf{A}=(H x, 0,0)$ in Eqs. (1) and (2) because we limit ourselves to the case when the effect of the selfinduced magnetic field is negligible. This is valid in the experimental situation because the width of the sample is much less than the characteristic length $\Lambda=\lambda(0)^{2} / d_{f}\left(d_{f}\right.$ is the thickness of the sample).

Strictly speaking Eq. (1) is valid only very close to the critical temperature (see estimates for different lowtemperature superconductors in Ref. 21). For example for bulk "clean" Al the validity of Eq. (1) was derived only for the range $\Delta T \sim 10^{-4} \mathrm{~K}$ near $T_{c}$. However our $\mathrm{Al}$ samples are in the "dirty" limit due to the small value of the mean path length $\ell$. As follows from Refs. 22 and 23 the relation between current density $j$, the absolute value of the order parameter $|\psi|$ and the momentum $p$ are quite close to the Ginzburg-Landau relation $j=p\left(1-p^{2}\right)=|\psi|^{2} p$ even when $T$ $\rightarrow 0$ for such samples. Besides, when we turn on the magnetic field and/or the transport current the density of states of quasiparticles differs from the Bardeen-Schriffer-Cooper dependence $^{24}$ and can become gapless ${ }^{24,25}$ for high enough magnetic fields and/or transport currents. In this case Eq. (1) should be valid at any temperature $T<T_{c}$ because they were actually derived in the gapless limit (with $\gamma=0)^{26}$ or for small value of the gap $\Delta(T) \ll k_{B} T_{c}{ }^{19}$

The actual value of $\gamma$ for $\mathrm{Al}$ should be about $10^{3}$ because the time $\tau_{E} \sim 10^{-8} \mathrm{~s}$ is quite large in this material. However, the use of such a large $\gamma$ value is important if we intend to compare quantitatively the theoretical and experimental val- 


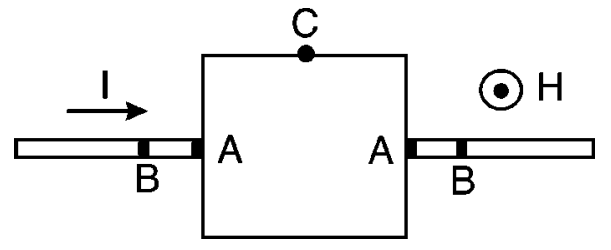

FIG. 4. Model geometry. Between points $A$ and $B$ phase slip centers appear at $H \lesssim H_{c 2}$. In point $C$ we trace out the dependence of $|\psi|$ on the magnetic field (see text below and Fig. 5).

ues for the critical current. As will be shown below there exist two critical currents which we call first $I_{c 1}$ and second $I_{c 2}$ critical currents. The first critical current is the current at which the sample goes to the nonresistive state and its value strongly depends on the value of the parameter $\gamma$ (in case of strong heat dissipation-see text below). The second critical current has the meaning of the current at which the superconducting state becomes unstable and it could be determined from a stability analysis of the stationary GinzburgLandau equations. It implies that the current $I_{c 2}$ does not depend on the $\gamma$ value which is the main reason why we are able to find quantitative agreement between theory and experiment for the position of the cusps in the $I_{c 2}(H)$ dependence (see below).

In our theoretical model we considered the geometry depicted in Fig. 4 which simulates the real experimental samples. To simplify our model we used linear contacts instead of the wedge shaped contacts used in the experiment. The main difference between them is that for wedge shaped contacts the order parameter is more suppressed in point $\mathrm{A}$ at low magnetic fields because the current density in this region is maximal (see Fig. 1). In order to inject the current in our system we used normal metal-superconductor boundary conditions at the end of the leads, i.e., $\psi=0$ and $-\nabla \varphi=j$. At the other boundaries we used the usual insulator-superconductor boundary conditions: $\left.(i \boldsymbol{\nabla}-\mathbf{A}) \psi\right|_{n}=0$ and $\left.\boldsymbol{\nabla} \varphi\right|_{n}=0$.

We also took into account the change of the local temperature in the sample in the resistive state by adding the heat diffusion equation to Eqs. (1) and (2)

$$
C_{e f f} \frac{\partial T}{\partial t}=K_{e f f} \Delta T+d_{f} j_{n}^{2} / \sigma_{n}-h\left(T-T_{0}\right),
$$

where $T_{0}$ is the bath temperature, $C_{e f f}=\left(D_{s} C_{s}+d_{f} C_{f}\right)$ is the effective heat capacity, $K_{e f f}=\left(D_{s} k_{s}+d_{f} k_{f}\right)$ is the effective heat conductivity coefficient, and the heat transfer coefficient $h$ $=k_{s} / D_{s}$ governs the heat removal from the sample. Here we used a model for the temperature distribution in thin superconducting films discussed in details in Ref. 27 and $C_{s}, C_{f}$, $k_{s}, k_{f}$ are the heat capacity and the heat conductivity of the substrate (subscript $s$ ) and film or sample (subscript $f$ ), respectively. In this model it is assumed that the thickness of the substrate and the film $D_{s}+d_{f}$ is much smaller than the

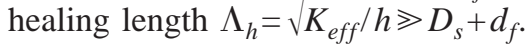

If heat removal is strong enough (large value for the coefficient $h$ ) we can neglect the effects due to the local change of the temperature. In the opposite case the results will quantitatively depend on the ratio between the healing length and the sample parameters (width of the square and the ratio between the value of the current density in the contacts and in the square). We chose our parameters in such a way that it optimizes the calculation time (small value of $C_{\text {eff }}$ ) and we considered cases of large, intermediate and small value of the coefficient $h$. In dimensionless units [the same as Eq. (1)] Eq. (3) may be written as follows

$$
\widetilde{C_{e f f}} \frac{\partial T}{\partial t}=\widetilde{K_{e f f}} \Delta T+j_{n}^{2}-\widetilde{h}\left(T-T_{0}\right)
$$

where $\widetilde{C_{e f f}}=\left(D_{s} C_{s} / d_{f}+C_{f}\right) T_{c} \sigma_{n} / \tau_{G L}(0) j_{0}^{2}, \quad \widetilde{K_{e f f}}=\left(D_{s} k_{s} / d_{f}\right.$ $\left.+k_{f}\right) T_{c} \sigma_{n} / \xi^{2}(0) j_{0}^{2}, \tilde{h}=h T_{c} \sigma_{n} / d_{f} j_{0}^{2}$ and the temperature is measured in units of $T_{c}$. If $D_{s} C_{s} / d_{f} \ll C_{f}$ and $D_{s} k_{s} / d_{f} \ll k_{f}$ we can use the Wiedemann-Franz law as an estimate for $C_{f}$ and $k_{f}$ and we obtain for $\widetilde{C_{\text {eff }}}=\pi^{3} / 48 \simeq 0.65$ and $\widetilde{K_{\text {eff }}}=\pi^{4} / 48 u^{2}$ $\simeq 0.06$ at a temperature close to $T_{c}$. These values should be considered only as a very rough estimate for the real magnitudes because normally the following inequalities are valid: $D_{s} C_{s} / d_{f} \gg C_{f}$ and $D_{s} k_{s} / d_{f} \gg k_{f}$. Because of the uncertainty in the actual values of $C_{s}$ and $k_{s}$ we used the following values: $\widetilde{C_{e f f}}=0.03$ (to optimize calculation time), $\widetilde{K_{e f f}}=0.06$, and $\tilde{h}$ $=2 \times 10^{-3}$ (which corresponds almost to full heat removal at $T=0.9), \tilde{h}=2 \times 10^{-4}$ (intermediate heat removal) and $\tilde{h}=2$ $\times 10^{-5}$ (weak heat removal) ${ }^{28}$ and a bath temperature of $T_{0}$ $=0.9$. We checked that our results only weakly depend on our choice of $\widetilde{C_{e f f}}$ and $\widetilde{K_{e f f}}$. As a boundary condition to Eq. (4) we take $\left.\nabla T\right|_{n}=0$ which means that heat is mainly transferred to the substrate. Only at the boundary between the normal metal and the superconductor we used boundary conditions with fixed temperature $T_{N S}=T_{0}$. The healing length is equal to $\Lambda_{h} \sim 11 \xi\left(T=0.9 T_{c}\right)$ for $\tilde{h}=2 \times 10^{-5}$ and is comparable to the size of the sample.

Although in our numerical calculations we used units normalized at $T=0$ we will discuss here mainly the situation for a bath temperature of $T_{0}=0.9 T_{c}$. Therefore, it is more convenient to express the different quantities in units normalized at $T=T_{0}$. For example, under $H_{c 2}$ we mean $H_{c 2}\left(T=0.9 T_{c}\right)$.

First we studied the behavior of our model geometry sample in an applied magnetic field with zero transport current. In Fig. 5 we present the dependence of the free energy of the square and the value of the order parameter in the center of the edge (i.e., at point $C$ in Fig. 4 ) as a function of the magnetic field. It turned out that for the chosen parameters (width of the square is $6 \xi$ and the size of the wires is $24 \xi \times \xi$ which are close to experimental values with $\xi$ $\simeq 0.333 \mu \mathrm{m}$ and $H_{c 2} \simeq 2.95 \mathrm{mT}$ ) no single quantum vortex state exists in the square and only surface superconductivity nucleates, i.e., the giant vortex state is present at $H$ $>0.8 H_{c 2}$. Superconductivity vanishes in the superconducting square at $H \geqslant 2.71 H_{c 2}$ which is much larger than the third critical field $H_{c 3}=1.69 \mathrm{H}_{c 2}$ of a flat infinite surface, which is a consequence of the shape and finite size of our system. By this value the vorticity in the system is equal to 10 and superconductivity may survive in the contacts up to much higher values of the magnetic field.

What will occur when we switch the transport current on? Let us consider first the situation when the heat removal is 


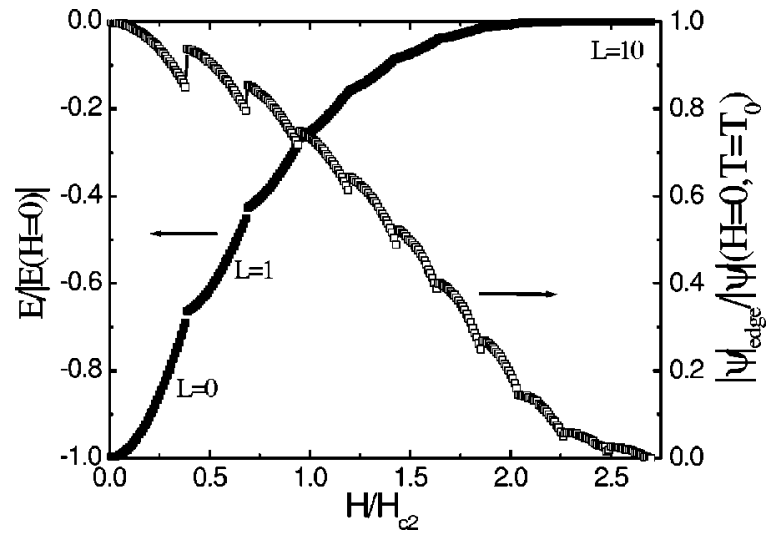

FIG. 5. Calculated dependence of the free energy (of the ground state) and the order parameter in the center of the edge of the superconducting square.

quite good and the temperature of the sample is equal to the bath temperature. Then up to some magnetic field $H^{*}\left(H^{*}\right.$ $\simeq 0.92 H_{c 2}$ for our parameters) the transition to the resistive state from the superconducting state occurs via the appearance of phase slip centers in the contacts [see Figs. 6(a) and 6(b)]. Current $I_{c 2}$ at which this occurs slightly depends on the applied field because the order parameter in the square depends on $H$ (see Fig. 5) due to the induced screening currents. Via proximity effects the variation in the order parameter in the square influences the order parameter in the contacts and hence the critical current for nucleation of the phase slip centers.

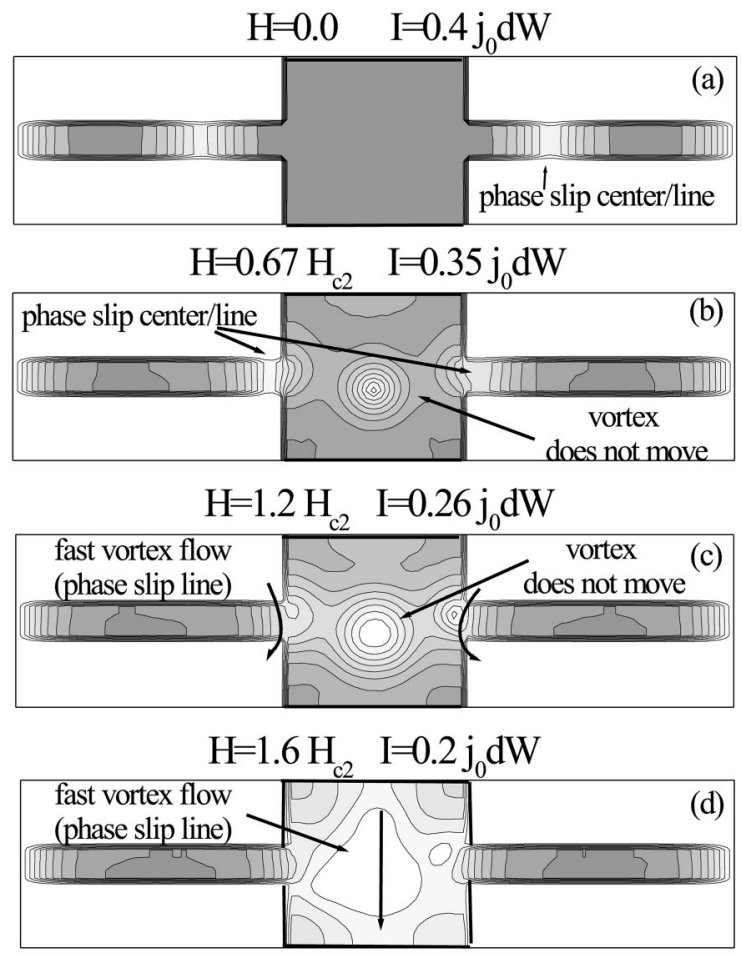

FIG. 6. Snapshots of the order parameter distribution (dark color corresponds to the maximal value of $\Psi$ and gray to the minimal one) in a superconducting square with attached leads at different magnetic fields and $I>I_{c 2}(H)$.
At higher magnetic fields the vortex flow regime starts at $I=I_{c 2}(H)$. At fields close to $H^{*}$ this regime switches to the phase slip process near the contacts [see Fig. 6(c)] while at fields higher than $H^{*}$ with further increase of the current it switches to the phase slip line regime in the square [or more exactly a line along which the vortices move very fast ${ }^{13}$ - see Fig. 6(d)]. It occurs because the order parameter is strongly suppressed in the square by the magnetic field while in the contacts its influence is less pronounced due to the small width of the contacts. The voltage exhibits a jump at the transition from slow flux flow to the phase slip line or center regime. The larger $H$ the smaller this jump in the voltage. ${ }^{13}$ The actual value of $H^{*}$, at which this change in the mechanism of destruction of superconducting state at $I=I_{c 2}(H)$ occurs, depends on the width of the contact. The narrower the contacts the higher the field at which flux flow in the square starts before the phase slip process occurs in the contacts.

We should note here that the position of the phase slip line in the contacts depends on the applied magnetic field. When the order parameter at the edge of the square decreases, the phase slip center approaches the square and vice versa. For the case of wedge contacts we do not expect such a behavior because in this case the order parameter is more suppressed around the narrowest point where the current density is maximal.

In Fig. 7 we present the $I-V$ characteristics calculated for different values of the magnetic field. For low magnetic fields and large heat transfer coefficient $\tilde{h}$ the hysteresis in the $I-V$ characteristics is an internal property of the phase slip process. ${ }^{4,7,13}$ At the current $I_{c 1}(H)$ the sample goes to the superconducting nonresistive state from the phase slip regime at fields less than some critical value $H^{* *}$ or from the flux flow regime for higher fields. The actual value of the field $H^{* *}$ depends on the value of the coefficient $\gamma$ and the width of the contact (see the above discussion for field $H^{*}$ ). With increasing $\gamma$, the minimal current at which the phase slip process is still possible, decreases and hence the field $H^{* *}$ increases, because the current at which the flux flow starts in the sample does not depend on the relaxation times of the superconducting condensate.

The magnetic field effects the hysteresis through the local suppression of the order parameter. ${ }^{7}$ This is the main origin of the decreasing and finally the disappearance of the hysteresis at the transition from the superconducting state or slow vortex flow regime to the phase slip regime at high magnetic fields. Another effect of the magnetic field is the slow increase of $I_{c 1}$ at low magnetic fields. The reason for this is the same as was found in Ref. 13-the nonuniform current density distribution in the contacts due to the applied magnetic field.

When the heat removal is not effective, then the $I-V$ characteristics have a different shape at low and intermediate magnetic fields. In Fig. 7 we present our results for two relatively small values of the heat transfer coefficient $\tilde{h}$. At low and intermediate magnetic fields the whole sample goes into the normal state (with $T>T_{c}$ or $T<T_{c}$ ) at the current $I=I_{c 2}(H)$ because of the large heat dissipation which is connected with the phase slip process. The value of that critical current is the same for any heat transfer coefficient $\tilde{h}$ due to 

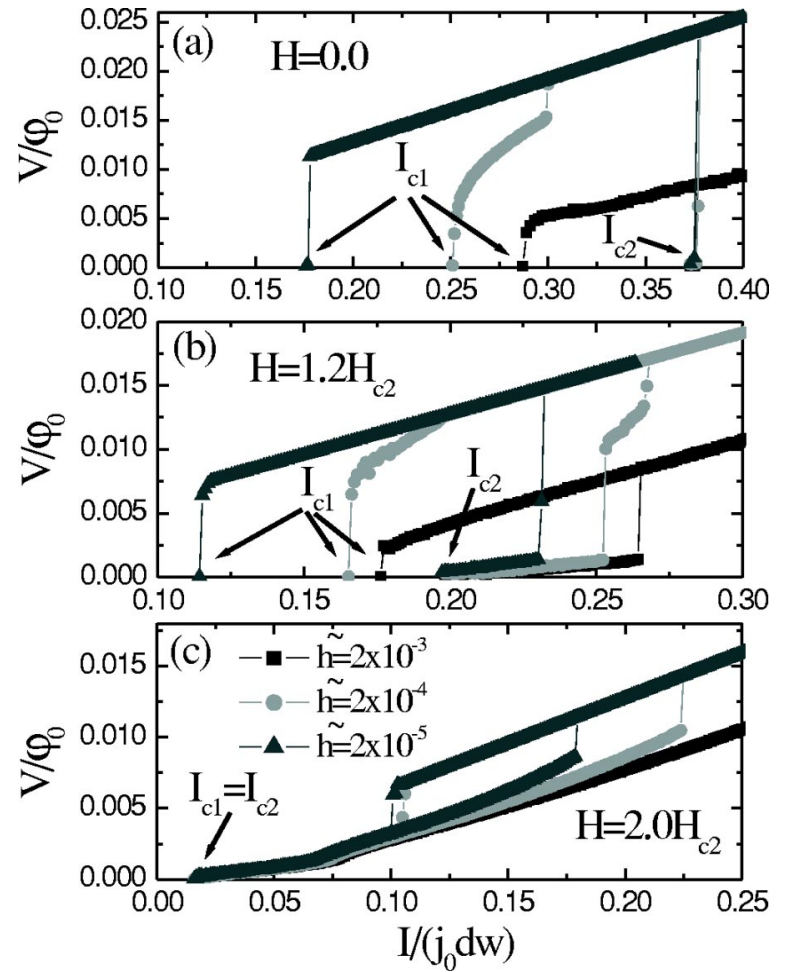

FIG. 7. Current-voltage characteristics of the superconducting square with contacts calculated for different heat removal coefficients $\tilde{h}$ and magnetic fields. Current is normalized by the value $j_{0} d w$, where $d$ and $w$ are the lead thickness and width, respectively.

the absence of heat dissipation in the "pure" superconducting state.

When we decrease the current, the temperature in the sample can become less than $T_{c}$ (see Fig. 8) while the sample will not go into the superconducting state because at this temperature the current in the sample is too high in order that superconductivity can sustain it. Only when the temperature in the sample becomes less than some critical temperature $T^{*}<T_{c}$ (which depends on the value of the heat transfer coefficient $\widetilde{h})$ then superconductivity starts to nucleate in the square in places where the current density is minimal (near the corners of the square). For this current, magnetic field, bath temperature and in the absence of local heating, the flux flow or phase slip processes are impossible (see Fig. 7 for high value of $\widetilde{h}$ ) and the sample should go to the superconducting state. But due to heat dissipation the actual temperature of the sample is still larger than $T_{0}$ (see Fig. 8). Consequently the sample is in the resistive state with a resistance less than the normal one. The range of currents for which such a process is possible depends on many parameters. For example, it increases with increasing magnetic field and decreasing heat transfer coefficient.

At high magnetic fields the critical currents $I_{c 2}$ and $I_{c 1}$ are quite small and even in the case of weak heat removal the $I-V$ characteristics resemble the ones with strong heat removal at low currents [Fig. 7(c)] due to weak heating [see Fig. 8(c)].

And finally in Fig. 9 we present the dependence of the currents $I_{c 2}$ and $I_{c 1}$ on the applied magnetic field for strong
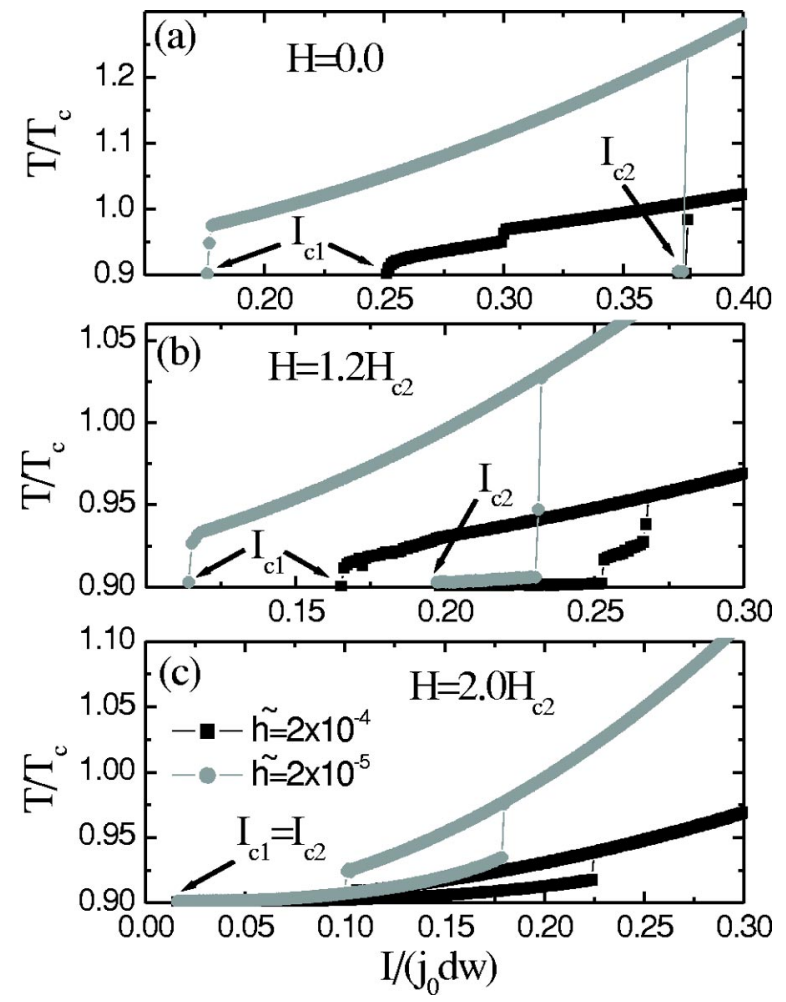

FIG. 8. Calculated dependence of the temperature of the superconducting square at different currents and magnetic fields. Results for $\tilde{h}=2 \times 10^{-3}$ are not presented due to quite small changes (less than $1 \%$ ) in the temperature.

and weak heat removal. There is a good quantitative agreement between the position of the cusps in the experimental and the theoretical dependencies of $I_{c 2}(H)$. We explain these cusps by abrupt changes in the vorticity and hence sharp changes in the order parameter distribution (see Fig. 5). The main difference between theory and experiment is in the amplitude of the variation of $I_{c 2}$ with $H$ (the theory predicts larger values) and in the value of this critical current. We believe that this disagreement originates mainly from the difference in the real shape of the attached contacts (Fig. 1) and the contacts used in our model (Fig. 4). In the experimental case there is a voltage lead which "strengthen" the superconducting property near the narrowest point (as it was shown in the quasi-one-dimensional limit in Ref. 29) and actually shifts the position of the phase slip center further from the square. Therefore, the effect of the variation of the order parameter in the square (with applied field) should be less pronounced on the phase slip process in the contacts. Variations in $I_{c 2}$ become stronger at $H \gtrsim H_{c 2}$ when the resistive regime starts from the vortex flow regime in the square and hence the effect of a change in the vorticity is more "visible."

We interpret the black right line in Fig. 3 as the line corresponding to the transition from the vortex flow to the phase slip line regime induced by increasing current at which a jump in the heat dissipation occurs. Because the heat removal is not effective, the square will become normal. Otherwise, it will be in the superconducting resistive state with a resistance close to the normal one. Here we would like to stress the following. In aluminum the decay length of the 
$\mathrm{I}(\mu \mathrm{A})$

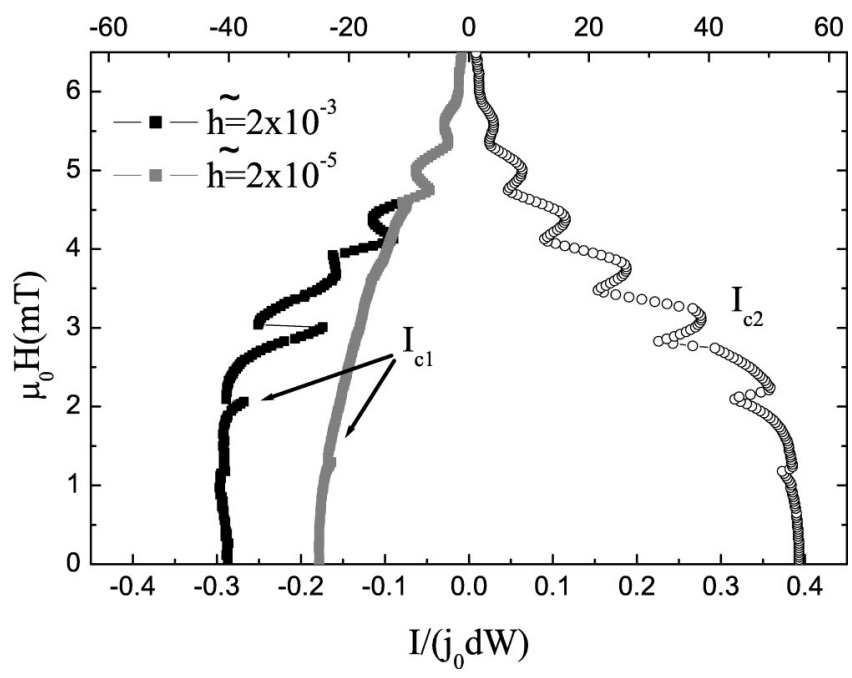

FIG. 9. Calculated dependence of the critical currents $I_{c 2}$ and $I_{c 1}$ (for $\tilde{h}=2 \times 10^{-3}$ and $\tilde{h}=2 \times 10^{-5}$ ) on the applied magnetic field. $I_{c 2}$ and $I_{c 1}$ coincide for $H \gtrsim 3.9 \mathrm{mT}\left(1.32 \mathrm{H}_{c 2}\right)$ at strong heat transfer $\left(\tilde{h}=2 \times 10^{-3}\right)$ and for $H \geqslant 4.6 \mathrm{mT}\left(1.56 H_{c 2}\right)$ at weak heat transfer $\left(\tilde{h}=2 \times 10^{-5}\right)$. There exists a nonmonotonous behavior both in the $I_{c 2}(H)$ and $I_{c 1}(H)$ dependencies which is connected with a change in the vorticity in the superconducting square.

charge imbalance $\Lambda_{Q}$ or, in other words, the region where the normal current density is finite near the phase slip center ${ }^{2}$ is quite large $^{30} \Lambda_{Q} \simeq 50 \mu \mathrm{m}$. The size of our sample is much less than $\Lambda_{Q}$. So in this case it is quite difficult to distinguish between the normal and the superconducting resistive state because the differential resistance would be the same and equal to the normal one (see Ref. 30 in Ref. 7).

With decreasing current (in absolute value) we cross the left black line (Fig. 3) and at low magnetic fields almost immediately enter the zero resistance state. Actually, the current $I_{c 1}$ even increases a little. The same increase of $I_{c 1}$ can be reproduced theoretically if we assume that these transitions occur due to the decay of the phase slip process in the contacts at strong heat removal (see Fig. 9).

A comparison with the experiment also shows that with increasing $H$ we cross the left black line and do not enter the zero resistance state. Thus we are probably in the vortex flow state but with the sample temperature larger than the bath temperature. The sample can be in the resistive state up to lower currents than it can be at the bath temperature (see Figs. 7 and 8 for small heat transfer coefficient).

At very high fields dissipation is not very important at currents close to the critical ones $I=I_{c 2}(H)=I_{c 1}(H)$ (due to their small value) and besides there is no "internal" hysteresis due to the phase slip process. As a result there is no hysteresis in the current when the nonzero resistance state appears and when a fast change in the resistance occurs in our sample.

\section{CONCLUSION}

Hysteresis in the current-voltage characteristics of superconducting wires, films or mesoscopic samples may appear due to heat dissipation or/and due to "intrinsic" hysteresis connected with the existence of phase slip lines or centers. We believe that in our measurements we have both types of hysteresis which are responsible for the observed effects. At low magnetic fields the sample enters the normal state due to the appearance of the phase slip process and a strong heat dissipation at the critical current $I_{c 2}$. Because of a discrete change of the vorticity in the superconducting square the order parameter changes abruptly at some values of $\mathrm{H}$ and it leads to cusps in the dependence of $I_{c 2}(H)$. At higher fields, instead of a high dissipative phase slip process, we have slow vortex motion and heat dissipation results in a weak effect on the $I-V$ characteristics for $I \gtrsim I_{c 2}(H)$.

When we decrease the current (at fixed value of the magnetic field) the sample goes first from the normal to the superconducting resistive state (left black line in Fig. 3) and then slow vortex flow starts in the superconducting strip (at high magnetic fields) or the phase slip process in the contacts (at low magnetic field). Because the temperature of the sample may be higher than the bath temperature, the current, at which the sample goes to the nonresistive state, may be smaller than $I_{c 2}$ even if the resistive state starts as a vortex flow. So actually we "need" heat dissipation to explain this effect.

One of the main results of our paper is that we show (by a self-consistent solution of the time-dependent GinzburgLandau equation and the heat diffusion equation) that heat dissipation does not necessarily lead to the destruction of superconductivity as it was supposed in a recent paper ${ }^{31}$ [in order to explain recent experiments on MoGe films (see references therein)]. In these samples the value of the coefficient $\tilde{h}$ is quite small due to the small value of the normal conductivity and both critical currents $I_{c 1}$ and $I_{c 2}$ are rather large because of the absence of the external magnetic field. In general, the situation may be more complicated when heating does not destroy superconductivity. In such a case, heating leads to additional complexity in the dynamics of the superconducting condensate due to the local heating of the sample.

Another result is, that by comparing the experimental and theoretical $I_{c 2}(H)$ dependence we may distinguish ${ }^{32}$ between the giant vortex and the single quantum vortex state which appear in the sample. It allows, in principle, to study experimentally the transformation between these two different configurations as a function of the shape, size of the sample, and external magnetic field.

\section{ACKNOWLEDGMENTS}

This work was supported by the Belgian Science Policy, GOA (University of Antwerp), the Research Fund K. U. Leuven GOA/2004/02, the ESF-programme VORTEX, and the Flemish Science Foundation (FWO-Vl). M.M. acknowledges support from the Institute for the Promotion of Innovation through Science and Technology in Flanders (IWTVlaanderen). 
*Electronic address: francois.peeters@ua.ac.be

${ }^{1}$ J. D. Meyer and G. Minnigerode, Phys. Lett. 38A, 529 (1972).

${ }^{2}$ W. J. Skocpol, M. R. Beasley, and M. Tinkham, J. Low Temp. Phys. 16, 145 (1974).

${ }^{3}$ G. J. Dolan and L. D. Jackel, Phys. Rev. Lett. 39, 1628 (1977).

${ }^{4}$ B. I. Ivlev and N. B. Kopnin, Adv. Phys. 33, 80 (1984).

${ }^{5}$ R. Tidecks, Current-Induced Nonequilibrium Phenomena in Quasi-One-Dimensional Superconductors (Springer, Berlin, 1990).

${ }^{6}$ M. Tinkham, Introduction to Superconductivity (McGraw-Hill, New York, 1996), Chap. 11.

${ }^{7}$ S. Michotte, S. Mátéfi-Tempfli, L. Piraux, D. Y. Vodolazov, and F. M. Peeters, Phys. Rev. B 69, 094512 (2004).

${ }^{8}$ F. S. Jelila, J.-P. Maneval, F.-R. Ladan, F. Chibane, A. Marie-deFicquelmont, L. Méchin, J.-C. Villégier, M. Aprili, and J. Lesueur, Phys. Rev. Lett. 81, 1933 (1998).

${ }^{9}$ S. Michotte, L. Piraux, F. Boyer, F. R. Ladan, and J. P. Maneval, Appl. Phys. Lett. 85, 3175 (2004).

${ }^{10}$ D. Y. Vodolazov, F. M. Peeters, L. Piraux, S. Mátéfi-Tempfli, and S. Michotte, Phys. Rev. Lett. 91, 157001 (2003).

${ }^{11}$ A. G. Sivakov, A. M. Glukhov, A. N. Omelyanchouk, Y. Koval, P. Müller, and A. V. Ustinov, Phys. Rev. Lett. 91, 267001 (2003).

${ }^{12}$ A. Andronov, I. Gordion, V. Kurin, I. Nefedov, and I. Shereshevsky, Physica C 213, 193 (1993).

${ }^{13}$ D. Y. Vodolazov, B. J. Baelus, and F. M. Peeters, Physica C 404, 400 (2004).

${ }^{14}$ N. Giordano, Phys. Rev. B 41, 6350 (1990); 43, 160 (1991); A. Bezryadin, C. N. Lau, and M. Tinkham, Nature (London) 404, 971 (2000); C. N. Lau, N. Markovic, M. Bockrath, A. Bezryadin, and M. Tinkham, Phys. Rev. Lett. 87, 217003 (2001).

${ }^{15}$ M. Morelle, G. Teniers, L. F. Chibotaru, A. Ceulemans, and V. V. Moshchalkov, Physica C 369, 351 (2002).

${ }^{16}$ M. Morelle, Y. Bruynseraede, and V. V. Moshchalkov, Phys. Status Solidi B 237, 365 (2003).

${ }^{17}$ V. M. Fomin, J. T. Devreese, and V. V. Moshchalkov, Europhys.
Lett. 42, 553 (1998); 46, 118 (1999).

${ }^{18}$ V. A. Schweigert and F. M. Peeters, Phys. Rev. B 60, 3084 (1999).

${ }^{19}$ L. Kramer and R. J. Watts-Tobin, Phys. Rev. Lett. 40, 1041 (1978).

${ }^{20}$ L. P. Gor'kov, Zh. Eksp. Teor. Fiz. 36, 1918 (1959) [Sov. Phys. JETP 9, 1364 (1959)]. We use a value which is different from the Bardeen-Cooper-Schrieffer value for $\Delta_{0}$ because Eq. (1) is only valid near $T_{c}$ where Delta ${ }_{T}=\Delta_{0}\left(1-T / T_{c}\right)^{1 / 2}$. Therefore, it should be considered only as an effective parameter rather than the actual value for the gap at $T=0 \Delta_{B C S} \simeq 1.74 k_{B} T_{c}$.

${ }^{21}$ R. J. Watts-Tobin, Y. Krähenbühl, and L. Kramer, J. Low Temp. Phys. 42, 459 (1981).

${ }^{22}$ J. Romijn, T. M. Klapwijk, M. J. Renne, and J. E. Mooij, Phys. Rev. B 26, 3648 (1982).

${ }^{23}$ J. Sanchez-Cañizares and F. Sols, J. Low Temp. Phys. 122, 11 (2001).

${ }^{24}$ Superconductivity, edited by R. D. Parks (Marcel Dekker, Inc., New York, 1969), Chap. 18, p. 1049.

${ }^{25}$ A. Anthore, H. Pothier, and D. Esteve, Phys. Rev. Lett. 90, 127001 (2003).

${ }^{26}$ A. Schmid and G. Schön, J. Low Temp. Phys. 20, 207 (1975).

${ }^{27}$ A. V. Gurevich and R. G. Mints, Rev. Mod. Phys. 59, 941 (1987).

${ }^{28}$ Our estimate of the experimental value for $\tilde{h}$ gives $\tilde{h} \simeq 2 \times 10^{-6}$ if we take the heat conductivity of our upper $\mathrm{SiO}_{2}$ substrate layer $d=500 \mathrm{~nm}$ at $\mathrm{T}=1.2 \mathrm{~K}$ as $k=6 \times 10^{-5} \mathrm{~W} / \mathrm{mK}$. Actually the heat conductivity of the main $\mathrm{Si}$ substrate is two orders of magnitude larger but in this case the condition $\Lambda_{h} \gg D_{s}+d_{f}$ is not satisfied. Therefore, in the experiment the value of $\tilde{h}$ should be in the range $10^{-3}-10^{-5}$.

${ }^{29}$ H. J. Fink and V. Grünfeld, Phys. Rev. B 31, 600 (1985).

${ }^{30}$ T. M. Klapwijk and J. E. Mooij, Phys. Lett. 57A, 97 (1976).

${ }^{31}$ M. Tinkham, J. U. Free, C. N. Lau, and N. Markovic, Phys. Rev. B 68, 134515 (2003).

${ }^{32}$ V. A. Schweigert, F. M. Peeters, and P. S. Deo, Phys. Rev. Lett. 81, 2783 (1998). 